\title{
Impact of uni- or multifocal perineural invasion in prostate cancer at radical prostatectomy
}

\author{
Alessandro Sciarra ${ }^{1}$, Martina Maggi ${ }^{1}$, Arianna Del Proposto ${ }^{1}$, Fabio Massimo Magliocca ${ }^{2}$, Antonio Ciardi ${ }^{2}$, \\ Valeria Panebianco ${ }^{2}$, Ettore De Berardinis ${ }^{1}$, Stefano Salciccia ${ }^{1}$, Giovanni Battista Di Pierro ${ }^{1}$, \\ Alessandro Gentilucci ${ }^{1}$, Alex M. Kasman ${ }^{3}$, Benjamin I. Chung ${ }^{3}$, Matteo Ferro ${ }^{4}$, Ottavio de Cobelli ${ }^{4}$, \\ Francesco Del Giudice ${ }^{1,3}$, Gian Maria Busetto ${ }^{1}$, Michele Gallucci ${ }^{1}$, Marco Frisenda ${ }^{1}$ \\ ${ }^{1}$ Department of Maternal-Infant and Urological Sciences, "Sapienza” Rome University, Policlinico Umberto I Hospital, Rome, Italy; ${ }^{2}$ Department \\ of Radiological, Oncological and Anatomopathological Sciences, "Sapienza" Rome University, Policlinico Umberto I Hospital, Rome, Italy; \\ ${ }^{3}$ Department of Urology, Stanford University School of Medicine, Stanford, CA, USA; ${ }^{4}$ Division of Urology, European Institute of Oncology (IEO), \\ Milan, Italy \\ Contributions: (I) Conception and design: A Sciarra, F Del Giudice, M Maggi, E De Berardinis, M Gallucci; (II) Administrative support: M Frisenda, \\ A Del Proposto, A Gentilucci, GB Di Pierro; (III) Provision of study materials or patients: A Sciarra, FM Magliocca, A Ciardi, V Panebianco, M \\ Gallucci; (IV) Collection and assembly of data: GM Busetto, M Frisenda, F Del Giudice, M Maggi, S Salciccia; (V) Data analysis and interpretation: \\ BI Chung, AM Kasman, M Frisenda, O de Cobelli; (VI) Manuscript writing: All authors; (VII) Final approval of manuscript: All authors. \\ Correspondence to: Francesco Del Giudice, MD. Department of Maternal-Infant and Urological Sciences, "Sapienza" Rome University, Policlinico \\ Umberto I Hospital, Rome, Italy; Department of Urology, Stanford University School of Medicine, 300 Pasteur Drive, Stanford, CA 94305-5118, \\ USA. Email: francesco.delgiudice@uniroma1.it; fdelgiu@stanford.edu.
}

Background: Aim of this study was to correlate perineural invasion (PNI) with other clinicalpathological parameters in terms of prognostic indicators in prostate cancer (PC) cases at the time of radical prostatectomy (RP).

Methods: Prospective study of 288 consecutive PC cases undergoing RP. PNI determination was performed either in biopsy or in RP specimens classifying as uni- and multifocal PNI. The median follow-up time was 22 (range, 6-36) months.

Results: At biopsy PNI was found in 34 (11.8\%) cases and in 202 (70.1\%) cases at the time of surgery. Among those identified at RP 133 (46.1\%) and 69 (23.9\%) cases had uni- and multi-PNI, respectively. Presence of PNI was significantly $(\mathrm{P}<0.05)$ correlated with unfavorable pathological parameters such higher stage and grade. The percentage of extracapsular extension in PNI negative RP specimens was $18.6 \% v s$. $60.4 \%$ of PNI positive specimens. However, the distribution of pathological staging and International Society of Urological Pathology (ISUP) grading did not vary according to whether PNI was uni- or multifocal. The risk of biochemical progression increased 2.3 times in PNI positive cases was significantly associated with the risk of biochemical progression $(\mathrm{r}=0.136 ; \mathrm{P}=0.04)$. However, at multivariate analysis PNI was not significantly associated with biochemical progression [hazard ratio (HR): 1.87, 95\% confidence interval (CI): 0.68-3.12; $\mathrm{P}=0.089$ ]. Within patients with intermediate risk disease, multifocal PNI was able to predict cases with lower mean time to biochemical and progression free survival (chi-square 5.95; $\mathrm{P}=0.04$ ).

Conclusions: PNI at biopsy is not a good predictor of the PNI incidence at the time of RP. PNI detection in surgical specimens may help stratify intermediate risk cases for the risk of biochemical progression.

Keywords: Prostatic neoplasm; perineural invasion (PNI); radical prostatectomy (RP)

Submitted Apr 06, 2020. Accepted for publication Sep 15, 2020.

doi: $10.21037 /$ tau-20-850

View this article at: http://dx.doi.org/10.21037/tau-20-850 


\section{Introduction}

Perineural invasion (PNI) is defined as "cancer tracking along or around nerves within the perineural space" (1). By invading the perineural space, tumor cells enter an environment that could provide pro-survival signals and the existence of PNI is a sign of impending tumor spread through lymphatic or vascular invasion and thus may be a poor prognostic sign (2). The relevance of this route of tumor extension is known for different neurotropic tumors such as pancreas, rectal and gastric cancer (3). PNI produces an interaction between nerves and neurotropic cancer cells, resulting in increased tumor growth; moreover, through the perineural space tumor cells may extend along tissue planes of least resistance. The result of these two aspects can improve tumor ability to progress locally and distally.

PNI is highly prevalent in prostate cancer (PC) as Lubig et al. (4) showed that, if carefully analyzed, it is found in $75 \%$ of radical prostatectomy (RP) specimens and in $25 \%$ of biopsies from patients with PC. Guidance from individual countries differs on whether PNI identification should be mandatory in pathologic description of PC cases (1). For example, the Royal College of Pathologists requires PNI description within pathology reports (5) whereas the College of American Pathologists suggests it may be optional (6).

Even though the concept of PNI is associated with a worse PC prognosis, its significance and diagnostic relevance remain controversial. Some studies have shown a higher risk of prostatic capsule invasion or lymphatic metastasis for PC cases with PNI (3,4,7-10) while other analyses have described PNI not being a significant predictor of prognosis in PC (1,11-14). Additionally, variability exists within the recognition of PNI as some authors have shown that PNI recognition may be improved with use of immunohistochemistry (4). Current pathologic reports are dichotomized as to whether PNI is present or not however the significance of this failing to capture complete circumferential or not infiltration of tumor cells into the nerve sheath that surround nerves is unknown $(1,4)$.

Therefore, we sought to correlate PNI with other clinical-pathological parameters in terms of prognostic indicators in a large series of PC cases at the time of RP. In particular, the aim of the present study was: to correlate PNI incidence at biopsy and at the time of RP; to correlate PNI incidence either with clinical parameters such as prostate-specific antigen (PSA) and multiparametric magnetic resonance imaging (mpMRI) Prostate Imaging-
Reporting and Data System (PI-RADS) score or with recognized pathologic prognostic factors such as Gleason score and local stage; to define the independent prognostic value of PNI in terms of biochemical and radiological progression.

We present the following article in accordance with the STROBE reporting checklist (available at http://dx.doi. org/10.21037/tau-20-850).

\section{Methods}

\section{Study population}

As part of the regular clinical practice in our institution, an internal committee approved (AR220172B18A8A89) this prospective study, where patients with a histological diagnosis of prostatic adenocarcinoma considered for RP as primary therapeutic option in our Urology Department were consecutively included in the analysis. The study was conducted in accordance with the Declaration of Helsinki (as revised in 2013). Informed consent was obtained from all the patients.

Inclusion criteria were: histological diagnosis of adenocarcinoma, no distant metastases at clinical staging (e.g., cT2-T3 N0 M0), determination of PNI in pathologic specimens from biopsy and RP, RP as primary treatment option. Exclusion criteria were: current active or prior treatment for other neoplasms, androgen deprivation therapy, chemotherapy, pelvic radiation therapy or treatment with other agents that could influence prostate tumor growth.

From January 2016 and January 2019, 288 consecutive patients with PC submitted to RP in our department corresponding to our inclusion and exclusion criteria were enclosed in our analysis.

\section{Clinical parameters}

All cases enclosed in the study were classified on the basis of clinical parameters described in Table 1. All cases were submitted to a standard random 14-core biopsy of the prostate. Before surgery, clinical staging and risk category (D'Amico and EAU classification) assessment were homogeneously performed using total PSA determination and imaging (MRI, CT, bone scan) following European Association of Urology (EAU) guidelines (15). From 2017 onward, patients underwent mpMRI with PI-RADS score determination (16) which was performed by an experienced 
Table 1 Patients characteristics in the whole population, and according to PNI status at surgery (P value chi-squared test)

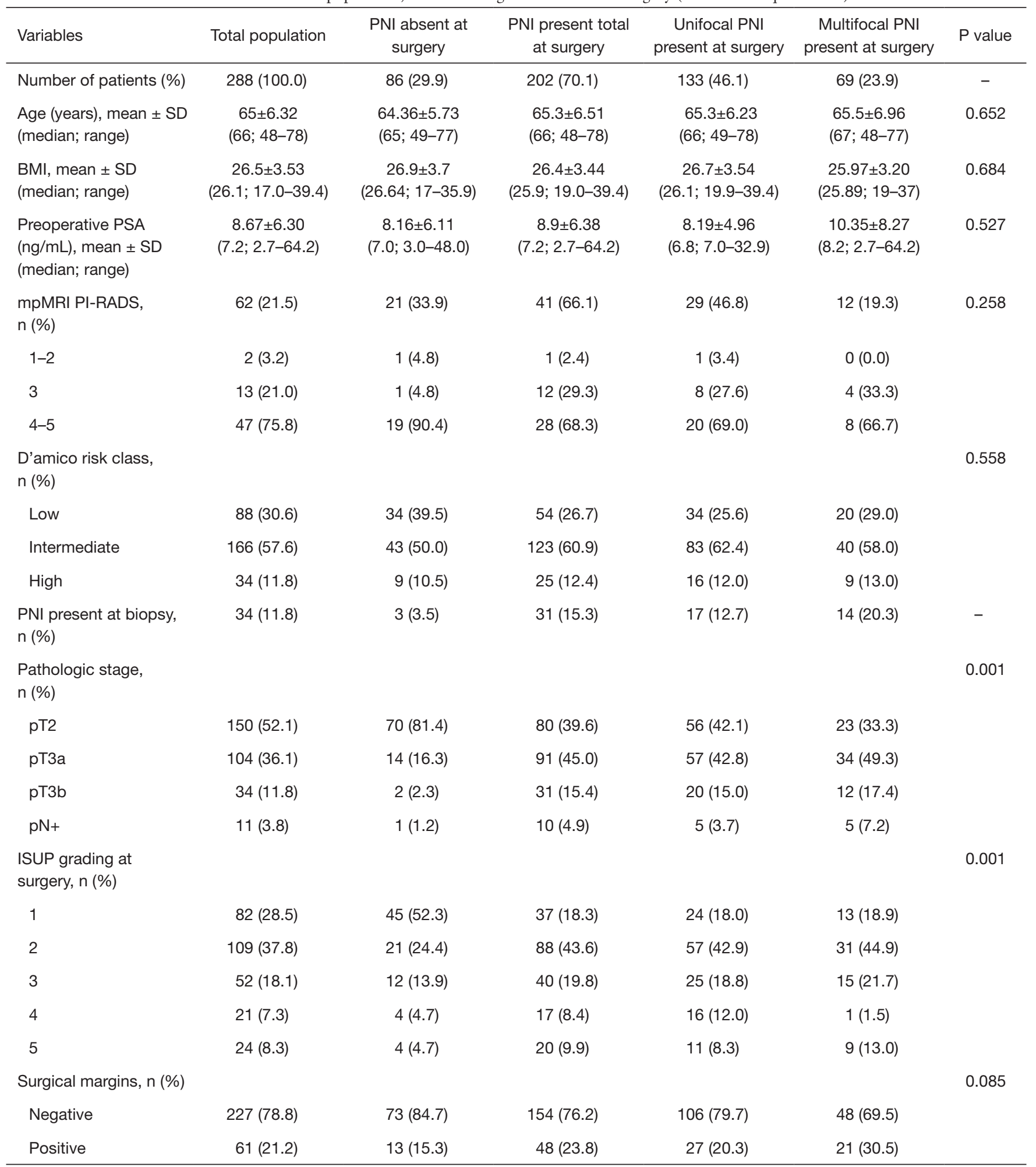

PNI, perineural invasion; SD, standard deviation; BMI, body mass index; PSA, prostate-specific antigen; mpMRI, multiparametric magnetic resonance imaging; PI-RADS, Prostate Imaging-Reporting and Data System; ISUP, International Society of Urological Pathology. 
genitourinary radiologist (VP). In cases with PI-RADS score of 3-5, targeted biopsy was performed in addition to the random biopsies as indicated by mpMRI. All patients underwent laparoscopic or robotic-assisted laparoscopic $\mathrm{RP}$ in our department following EAU guidelines for indications (15). After surgery, all patients were followed at regular intervals per EAU guidelines and time to biochemical recurrence (confirmed total PSA progression over $0.2 \mathrm{ng} / \mathrm{mL}$ ), radiological progression and overall survival were noted during follow up (15).

\section{Pathologic evaluation}

All histological specimens from prostatic biopsy and RP were examined by two experienced genitourinary pathologists (FMM and AC). Gleason score and International Society of Urological Pathology (ISUP) grading at biopsy and at surgery, pathologic staging using TNM classification and surgical margin status were defined in all cases. PNI determination was performed either in biopsy or in surgical specimens for all patients. Tissue specimens were stained with standard immunohistochemical techniques (avidinbiotin-peroxidase complex) using antibody to $\mathrm{S} 100$ protein to delineate better anatomical features of small nerves $(1,4)$. The nerve course was traced in ink on the slides, related to the prostate capsule and surrounding fascia in different regions of the prostate. The findings were translated into a three-dimensional reconstruction of the nerve course from the neurovascular bundle through the prostate capsule and into the parenchyma using standard methods of serial micro-reconstruction. In all cases the slides were searched for areas of perineural space invasion by carcinoma and the involved nerves were marked in ink. In equivocal areas the presence of nerve was confirmed with S100 staining. For each area of capsule penetration, the location of the area on the capsule was determined. In each high-power field of interest, PNI was defined positive as the presence of tumor cells in direct contact with nerve tissue, either partially or completely surrounding nerves. On the basis of this parameter, cases were classified as positive or negative (nerves without immediate epithelial tumor cell contact) for PNI. PNI was classified as uni- or multifocal.

\section{Statistical analysis}

For statistical evaluation SPSS Statistics program was used. Descriptive statistical methods such as number of cases, mean \pm standard deviation $(\mathrm{SD})$, median and range were used. For the comparison of quantitative data and pairwise intergroup comparisons of variables Mann Whitney test was performed. For comparison of qualitative data Fisher's Exact test, chi-square test and $\mathrm{McNemar}$ test were used. Pearson correlation analysis was also performed. Univariate and multivariate Cox proportional analysis considering clinical and pathological parameters were used. KaplanMeier survival curves related to PNI status and to the clinical outcomes were obtained. Statistical significance was evaluated at $\mathrm{P}<0.05$.

\section{Results}

Baseline characteristics of the 288 cases included in our analysis are described in Table 1. The median follow-up time after surgery was 22 (mean $\pm \mathrm{SD}, 21.8 \pm 12.4$; range, 6-36) months. At biopsy, PNI was found in 34 (11.8\%) cases versus at surgery in $202(70.1 \%)$ cases, among which $133(46.1 \%)$ and 69 (23.9\%) cases had unifocal PNI and multifocal PNI, respectively.

\section{Correlation among PNI incidence and clinical parameters}

Clinical parameters such as age, body mass index (BMI) and preoperative PSA were not significantly $(\mathrm{P}>0.50)$ different between cases with and without PNI (Table 1). Additionally, the distribution of risk classes did not significantly differ with regard to PNI status ( $\mathrm{P}=0.558)$. Pearson correlation analysis showed no significant correlation between PNI status and risk classes $(\mathrm{r}=0.082 ; \mathrm{P}=0.121)$ or $\mathrm{mpMRI}$ PIRADS score distribution $(\mathrm{r}=-0.162 ; \mathrm{P}=0.216)$ (Table 2). There were a higher percentage of PI-RADS 4-5 cases which was independent of PNI status (Table 1).

\section{Correlation among PNI incidence and pathological parameters}

Presence of PNI was significantly $(\mathrm{P}<0.05)$ correlated with unfavorable pathological parameters such as higher $\mathrm{T}$ stage and grade. In particular the percentage of localized PC at surgery without PNI was $81.4 \%$ vs. $39.6 \%$ in cases with PNI, whereas the percentage of extracapsular PC was $18.6 \%$ when PNI was absent versus $60.4 \%$ when it was present (Table 1, Figure 1A). The percentage of ISUP $1 \mathrm{PC}$ was $52.3 \%$ in PNI negative specimens compared to $18.3 \%$ in PNI positive specimens (Table 1, Figure 1B). The percentage of positive surgical margins was slightly but not significantly higher in PNI positive (23.8\%) than in PNI 
Table 2 Correlation coefficients among PNI status and other clinical and pathological parameters (Pearson coefficient)

\begin{tabular}{lcc}
\hline Correlation & Coefficient & P value \\
\hline PNI-risk class & 0.082 & 0.121 \\
PNI-mpMRI PI-RADS & -0.162 & 0.216 \\
PNI-pathological stage & 0.352 & $<0.01$ \\
PNI-pathological grading & 0.195 & 0.03 \\
PNI-Surgical margins & 0.135 & 0.09 \\
PNI-biochemical progression & 0.136 & 0.04 \\
PNI-radiological progression & 0.078 & 0.223 \\
\hline
\end{tabular}

$\mathrm{PNI}$, perineural invasion; mpMRI, multiparametric magnetic resonance imaging; PI-RADS, Prostate Imaging-Reporting and Data System.

negative $(15.3 \%)$ cases $(\mathrm{P}=0.275)$ (Table 1, Figure 1C). In $\mathrm{PNI}$ positive cases, the distribution of pathological staging and ISUP grading did not vary according to the uni- or multifocality of PNI (Table 1). Pearson correlation analysis showed a significant correlation between PNI status and pathological stage $(\mathrm{r}=0.352 ; \mathrm{P}<0.01)$ or ISUP grading $(\mathrm{r}=0.195 ; \mathrm{P}=0.03)$ (Table 2).

\section{PNI and clinical outcomes}

During postoperative follow-up $14.9 \%$ of our population developed a biochemical progression while $5.9 \%$ progressed radiologically with no deaths. PNI positivity was significantly correlated with biochemical progression $(\mathrm{r}=0.136 ; \mathrm{P}=0.04)$ (Table 2). In comparison to those without PNI, cases with PNI had a higher rate of biochemical relapse $(17.8 \%$ vs. $8.1 \%)$, radiological progression $(6.9 \%$ vs. $3.5 \%)$ and local recurrence ( $4.0 \%$ vs. $2.3 \%$ ), while lymphnode or distant progression rates were low and independent of PNI status (Table 3). Moreover, in PNI positive cases the percentage of biochemical progression increased if PNI was multifocal versus unifocal (21.7\% vs. 15.7\%). Kaplan-Meier survival analysis showed that mean biochemical progression free survival was significantly (chi-square 6.491; $\mathrm{P}=0.039$ ) lower in cases with PNI (unifocal: $30.42 \pm 1.18$ months; multifocal: $28.13 \pm 1.91$ months) when compared with those without PNI (33.26 \pm 1.16 months) (Figure 2, Table 3). On the contrary, no significant (chi-square 1.83; $\mathrm{P}=0.40$ ) differences in terms of mean radiological progression free survival were found according to PNI status $(35.03 \pm 0.83$; $34.36 \pm 0.69 ; 33.83 \pm 1.13$ months respectively in absent, uni- and multifocal PNI) (Figure 2, Table 3). In the intermediate risk class, but not in the low and high risk classes (Figure 3), PNI was able to predict cases with lower mean biochemical progression free survival when compared to cases without PNI. In particular, within the intermediate risk class, cases with multifocal PNI showed significantly (chi-square 5.95; $\mathrm{P}=0.04$ ) lower mean biochemical progression free survival $\{32.84 \pm 1.74$ [95\% confidence interval (CI): 29.43-36.24] months\} when compared to cases without PNI $[26.99 \pm 2.70$ (95\% CI: 21.68-32.29) months].

\section{Cox regression analysis for PNI independent prognostic value}

Cox regression analysis to identify predictors of biochemical progression in our population is displayed in Table 4. Univariate analysis had shown that PNI was a significant predictor of biochemical progression with a 2.33 -fold higher risk when compared with cases without PNI [hazard ratio (HR): 2.33, 95\% CI: 1.03-5.25; $\mathrm{P}=0.040$ ]. Other significant predictors of biochemical progression at univariate analysis were pathological stage, risk class, ISUP grading and surgical margins status. At multivariate analysis, after adjusting for pathological stage, grading and surgical margins, PNI was no longer a statistically significant predictor of biochemical progression (HR: 1.87, 95\% CI: $0.68-3.12 ; \mathrm{P}=0.089)$.

\section{Discussion}

PNI is a recognized route of tumor dissemination associated with unfavorable outcomes in many malignancies (17). The interaction between nerves and malignant cells can result in cancer progression, invasion and motility. Although associated with unfavorable clinical outcomes in several neoplasms, concrete guidelines concerning PNI in PC management remain absent. Examination for PNI in PC cases is also time consuming and spending time to examine every case for PNI when adequate risk stratification and clinical guidelines are missing may be unjustified $(1,4)$. This is particularly true when looking for PNI within prostate biopsy specimens. The presence or absence of nerves in a biopsy is not regularly recorded and therefore a negative result may indicate that no nerves were identified. The majority of studies on PNI in PC examine patients treated via $\mathrm{RP}$ and thus utilize whole prostatectomy specimens rather than biopsy cores $(7,9-14)$. Moreira et al. (18) 


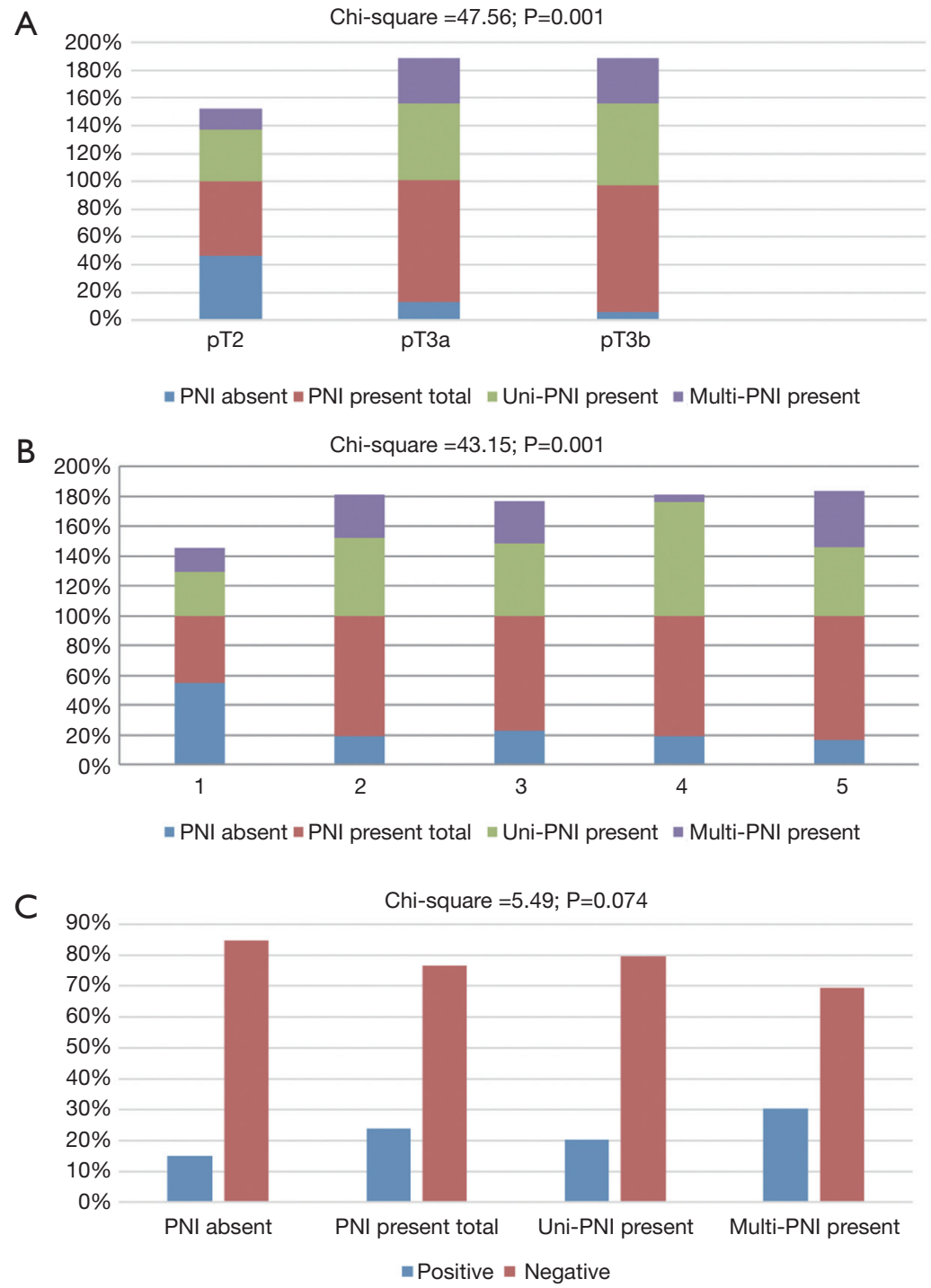

Figure 1 Bar-chart showing the percentage of patients with PNI (absent, present, uni- or multifocal) at surgery according to: (A) pathological stage; (B) ISUP grading; (C) surgical margins. Chi-square analysis. PNI, perineural invasion; ISUP, International Society of Urological Pathology.

investigated 302 patients on active surveillance and showed that PNI at biopsy was associated with a $73 \%$ chance of clinical progression after 2 years. In our experience on 288 patients undergoing $\mathrm{RP}$, prostate biopsy was not a useful method to stratify cases on the basis of PNI, since there was a weak correlation between biopsy and RP findings [at biopsy PNI was found in only $34(11.8 \%)$ cases, whereas at surgery in $202(70.1 \%)$ cases].

Another relevant point is the quantitative assessment of PNI at surgery. Considering the high prevalence, PNI is frequently detected in $\mathrm{RP}$ examinations and therefore a stratification of PNI positive cases on the basis of quantitative parameters may provide a better indicator of progression risk. Maru et al. (19) measured the diameter of PNI in RP specimens and found that increased diameter was strongly associated with biochemical progression. Saeter et al. (3) showed that increasing amount of PNI was strongly associated with higher pathological stage and risk of biochemical progression. In clinical practice several pathologists prefer to quantitatively define PNI at RP simply as unifocal versus multifocal. Sun et al. (7), analyzed 265 RP specimens and showed that patients with multifocal 
Table 3 Clinical outcomes at follow-up after surgery according to PNI results at RP (log-rank Mantel-Cox and Breslow-Wilcoxon analysis of chisquare)

\begin{tabular}{|c|c|c|c|c|}
\hline Outcome & PNI absent & Unifocal PNI & Multifocal PNI & $P$ value \\
\hline Local recurrence, n (\%) & $2 / 86(2.3)$ & 4/133 (3.0) & $4 / 69(5.8)$ & $1.77(0.08)$ \\
\hline Distant metastases, n (\%) & $0(0.0)$ & $1 / 133(0.7)$ & $1 / 69(1.4)$ & $0.78(0.56)$ \\
\hline
\end{tabular}

$\mathrm{PNI}$, perineural invasion; RP, radical prostatectomy; SD, standard deviation; $\mathrm{Cl}$, confidence interval; $\mathrm{n}$, number.
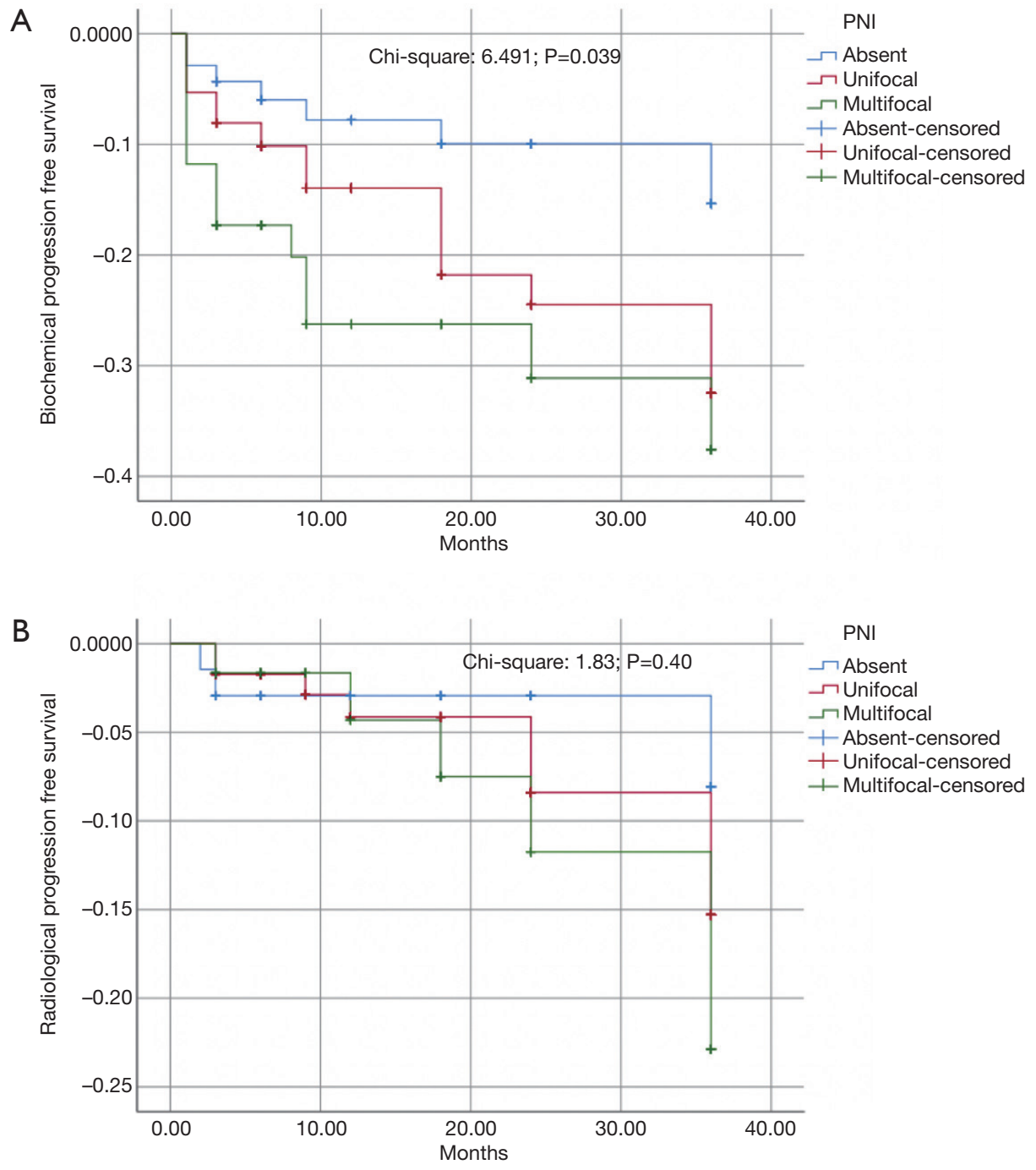

Figure 2 Kaplan-Meier curve showing logarithmic biochemical (A) and radiological (B) progression-free survival following RP according to PNI absent, present, uni- or multifocal (log-rank Mantel-Cox: biochemical progression: chi-square 6.491, $\mathrm{P}=0.039$; radiological progression: chi-square $1.83, \mathrm{P}=0.40)$. RP, radical prostatectomy; $\mathrm{PNI}$, perineural invasion. 
A

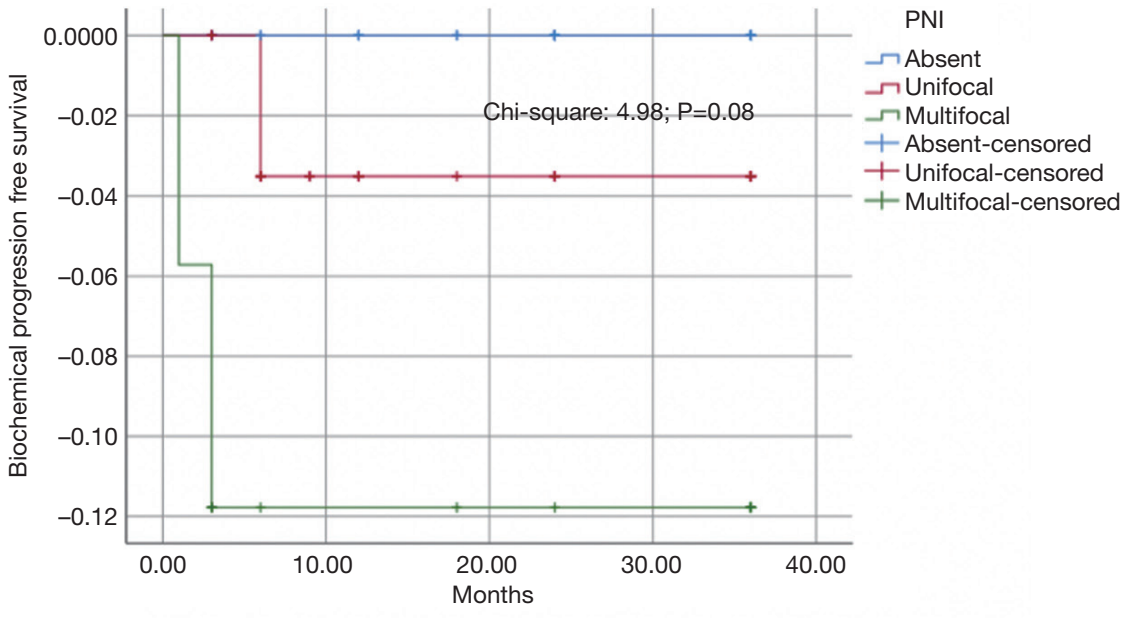

B

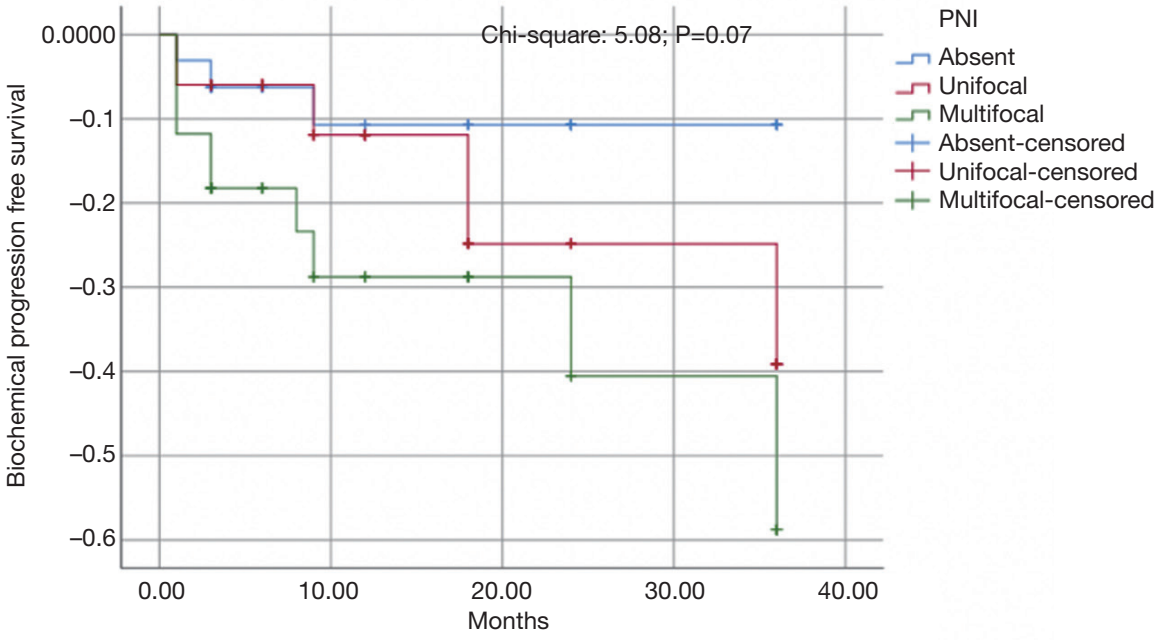

C

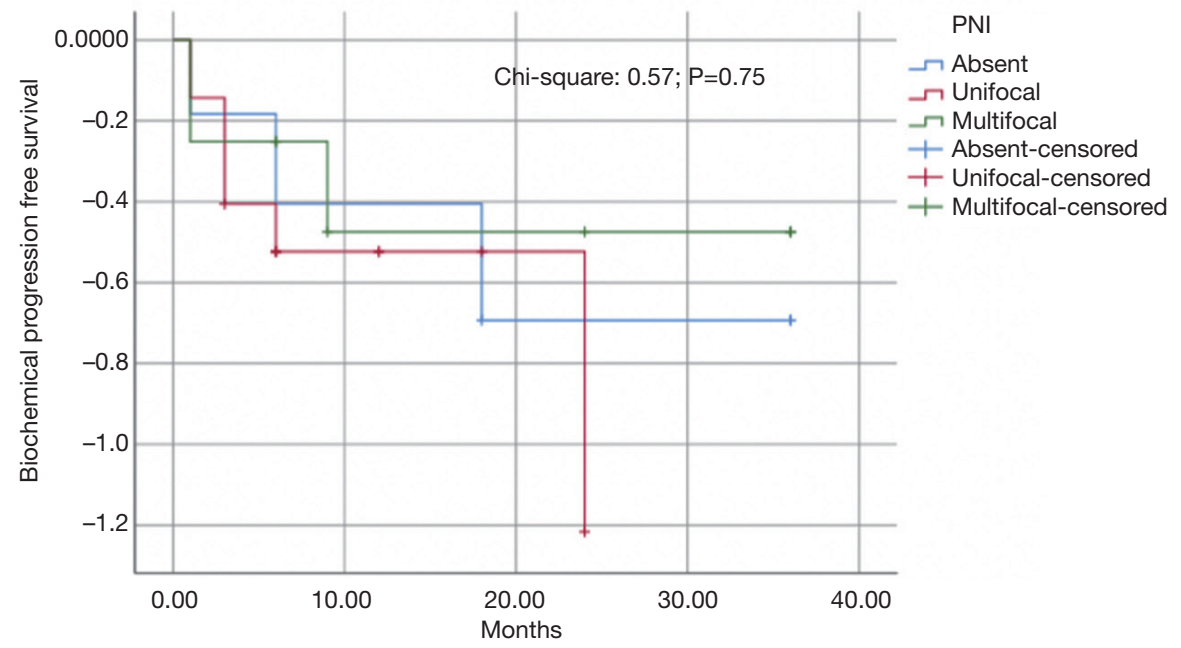

Figure 3 Kaplan-Meier curve showing logarithmic biochemical progression-free survival following RP in low (A), intermediate (B) and high (C) risk cases according to PNI absent, present, uni- or multifocal (log-rank Mantel-Cox: low risk: chi-square 4.98, $\mathrm{P}=0.08$; intermediate risk: chi-square $5.08, \mathrm{P}=0.07$; high risk: chi-square $0.57, \mathrm{P}=0.75$ ). $\mathrm{RP}$, radical prostatectomy; PNI, perineural invasion. 
Table 4 Cox regression analysis for the identification of the pathological predictors for biochemical progression after surgery (HR, $95 \%$ CI and $\mathrm{P}$ value)

\begin{tabular}{|c|c|c|c|c|c|c|}
\hline Variables & \multicolumn{3}{|c|}{ Univariate } & \multicolumn{3}{|c|}{ Multivariate } \\
\hline \multicolumn{7}{|l|}{ Risk class } \\
\hline Low & 1.0 & - & - & 1.0 & - & - \\
\hline Intermediate & 5.88 & $1.78-19.48$ & 0.004 & 3.06 & $0.88-10.61$ & 0.078 \\
\hline \multicolumn{7}{|c|}{ Pathologic stage } \\
\hline pT2 & 1.0 & - & - & 1.0 & - & - \\
\hline рT3a & 3.11 & $1.38-6.99$ & 0.006 & 1.90 & $0.82-4.41$ & 0.133 \\
\hline pT3b & 10.09 & $4.48-22.70$ & $<0.001$ & 4.38 & $1.84-10.43$ & 0.001 \\
\hline 2 & 2.77 & $0.75-10.26$ & 0.126 & 1.26 & $0.43-3.58$ & 0.356 \\
\hline 3 & 9.08 & $2.58-31.96$ & 0.001 & 4.04 & $2.15-6.43$ & 0.035 \\
\hline 4 & 11.45 & $3.03-43.22$ & $<0.001$ & 6.75 & $3.28-18.25$ & 0.001 \\
\hline 5 & 14.92 & $4.10-54.30$ & $<0.001$ & 8.43 & $4.47-27.76$ & $<0.001$ \\
\hline \multicolumn{7}{|l|}{ PNI at surgery } \\
\hline Absent & 1.00 & - & - & 1.00 & - & - \\
\hline Present & 2.33 & $1.03-5.25$ & 0.040 & 1.87 & $0.68-3.12$ & 0.089 \\
\hline \multicolumn{7}{|c|}{ Surgical margins } \\
\hline
\end{tabular}

$\mathrm{HR}$, hazard ratio; Cl, confidence interval; ISUP, International Society of Urological Pathology; PNI, perineural invasion.

PNI had a higher rate of extracapsular disease (pT3b) and it was an independent predictor of biochemical progression.

In our experience, the presence of PNI at RP was significantly $(\mathrm{P}<0.05)$ correlated with unfavorable pathological parameters such as local stage and grading but not with the risk of positive surgical margins. In particular the percentage of localized PC at surgery was $81.4 \%$ in PNI negative specimens and $39.6 \%$ in PNI positive specimens, whereas the percentage of extracapsular PC was $18.6 \%$ in PNI negative specimens and $60.4 \%$ in PNI positive specimens. The percentage of ISUP 1 PC was $52.3 \%$ in PNI negative cases and $18.3 \%$ in PNI positive cases. However, in PNI positive cases, the distribution of pathological staging and ISUP grading did not vary according to uni- or multifocality of PNI.
In our population the risk of biochemical progression increased 2.3 times in PNI cases when compared to negative cases and multifocality of PNI was significantly associated with the risk of biochemical progression $(\mathrm{r}=0.136 ; \mathrm{P}=0.04)$ with rates increasing from $15.7 \%$ in unifocal to $21.7 \%$ in multifocal versus only $8.1 \%$ in negative cases. Moreover, in PNI positive cases the percentage of local recurrence increased $(4.0 \%$ vs. $2.3 \%)$ while lymph-node or distant progression rates were very low and independent of PNI status. However, at a multivariate analysis, after adjusting for pathological stage, grading and surgical margins, PNI was not a statistically significant predictor of biochemical progression (HR: 1.87, 95\% CI: 0.68-3.12; $\mathrm{P}=0.089$ ).

Another clinically relevant aspect of PNI presence is the possibility to better stratify cases in the intermediate 
risk class in terms of risk of progression after RP. In our population, in the intermediate risk class PNI and in particular multifocal PNI, was able to identify cases with lower mean biochemical progression free survival when compared to cases without PNI (chi-square 5.95; $\mathrm{P}=0.04$ ).

There are several limitations of the present study that warrant mention. One, we identified a low rate of radiological progression after surgery and our postoperative follow-up time is short thus limiting the ability to examine cancer specific mortality. However, our population represent a standard clinical setting of patients submitted to $\mathrm{RP}$ in which correct indications can produce good results in terms of rates of biochemical and radiological progression.

\section{Conclusions}

Currently, many experienced urologists state that PNI is not crucial to have on the pathological report which contributes to the low reporting of PNI and limits the evaluation of its true clinical significance $(7,20)$. In our experience PNI rate at biopsy is not a good predictor of PNI incidence at the time of RP. At RP, PNI was significantly associated with unfavorable pathological parameters such as an extracapsular extension and high ISUP neoplasm but not with the risk of positive surgical margins. Though the risk of biochemical progression increased 2.3 times in cases with PNI, after adjusting for pathological stage, grading and surgical margins, PNI was no longer a statistically significant predictor of progression.

\section{Acknowledgments}

Funding: None.

\section{Footnote}

Reporting Checklist: The authors have completed the STROBE reporting checklist. Available at http://dx.doi. org/10.21037/tau-20-850

Data Sharing Statement: Available at http://dx.doi. org/10.21037/tau-20-850

Peer Review File: Available at http://dx.doi.org/10.21037/ tau-20-850

Conflicts of Interest: All authors have completed the ICMJE uniform disclosure form (available at http://dx.doi. org/10.21037/tau-20-850). The authors have no conflicts of interest to declare.

Ethical Statement: The authors are accountable for all aspects of the work in ensuring that questions related to the accuracy or integrity of any part of the work are appropriately investigated and resolved. The study was conducted in accordance with the Declaration of Helsinki (as revised in 2013) and as part of the regular clinical practice in our institution, an internal committee approved (AR220172B18A8A89) this prospective study. Informed consent was obtained from all the patients.

Open Access Statement: This is an Open Access article distributed in accordance with the Creative Commons Attribution-NonCommercial-NoDerivs 4.0 International License (CC BY-NC-ND 4.0), which permits the noncommercial replication and distribution of the article with the strict proviso that no changes or edits are made and the original work is properly cited (including links to both the formal publication through the relevant DOI and the license). See: https://creativecommons.org/licenses/by-nc-nd/4.0/.

\section{References}

1. Ahmad AS, Parameshwaran V, Beltran L, et al. Should reporting of perineural invasion and extraprostatic extension be mandatory in prostate cancer biopsy? correlation with outcome in biopsy cases treated conservatively. Oncotarget 2018;9:20555-62.

2. Ayala GE, Dai H, Tahir SA, et al. Stroma antiapoptotic paracrine loop in perineural invasion of prostatic carcinoma. Cancer Res 2006;66:5159-64.

3. Saeter T, Bogaard M, Vlatkovic L, et al. The relationship between perineural invasion, tumor grade, reactive stroma and prostate cancer specific mortality: a clinicopathologic study on a population-based cohort. Prostate 2016;76:207-14.

4. Lubig S, Thiesler T, Müller S, et al. Quantitative perineural invasion is a prognostic marker in prostate cancer. Pathology 2018;50:298-304.

5. Prostate dataset proformas. 2016. Available online: https://www.rcpath.org/resourceLibrary/g048prostatedatasetproformas-jun16-docx.html

6. College of American Pathologists. Protocol for the examination of specimens from patients with carcinoma of the prostate gland. 2017. Available online: https:// documents.cap.org/protocols/cp-prostate-2017-v4020.pdf 
7. Sun G, Huang R, Zhang X, et al. The impact of multifocal perineural invasion on biochemical recurrence and timing of adjuvant androgen deprivation therapy in high risk prostate cancer following radical prostatectomy. Prostate 2017;77:1279-87.

8. Harnden P, Shelley MD, Clements H, et al. The prognostic significance of perineural invasion in prostatic cancer biopsies. Cancer 2007;109:13-24.

9. Meng $\mathrm{Y}$, Liao $\mathrm{YB}, \mathrm{Xu} \mathrm{P}$, et al. Perineural invasion is an independent predictor of biochemical recurrence of prostate cancer after local treatment. Int J Clin Exp Med 2015;8:13267-74.

10. Zhang LJ, Wu B, Zha ZL, et al. Perineural invasion as an independent predictor of biochemical recurrence in prostate cancer following radical prostatectomy or radiotherapy: a systematic review and meta-analysis. BMC Urol 2018;18:5.

11. Celik S, Bozkurt O, Demir O, et al. Effects of perineural invasion in prostate needle biopsy on tumor grade and biochemical recurrence rates after radical prostatectomy. Kaohsiung J Med Sci 2018;34:385-90.

12. Noale M, Maggi S, Artibani W, et al. Pros-IT CNR: an Italian prostate cancer monitoring project. Aging Clin Exp Res 2017;29:165-72.

13. Antonelli A, Palumbo C, Noale M, et al. Overview of potential determinants of radical prostatectomy versus radiation therapy in management of clinically localized prostate cancer: results from an Italian prospective observational study. Minerva Urol Nefrol 2020;72:595-604.

Cite this article as: Sciarra A, Maggi M, Del Proposto A, Magliocca FM, Ciardi A, Panebianco V, De Berardinis E, Salciccia S, Di Pierro GB, Gentilucci A, Kasman AM, Chung BI, Ferro M, de Cobelli O, Del Giudice F, Busetto GM, Gallucci M, Frisenda M. Impact of uni- or multifocal perineural invasion in prostate cancer at radical prostatectomy. Transl Androl Urol 2021;10(1):66-76. doi: 10.21037/tau-20-850
14. Niroomand H, Nowroozi M, Ayati M, et al. Relationship between perineural invasion in prostate needle biopsy specimens and pathologic staging after radical prostatectomy. Nephrourol Mon 2016;8:e36022.

15. Mottet N, Cornford P, van den Bergh RCN, et al. Prostate Cancer: European Association of Urology (EAU) guidelines. 2019. Available online: https://uroweb.org/ guideline/prostate-cancer/

16. Maggi M, Panebianco V, Mosca M, et al. Prostate Imaging Reporting and Data System 3 category cases at multiparametric magnetic resonance for prostate cancer: a systematic review and meta-analysis. Eur Urol Focus 2020;6:463-78.

17. Saeter T, Vlatkovic L, Waaler G, et al. The prognostic value of reactive stroma on prostate needle biopsy: a population-based study. Prostate 2015;75:662-71.

18. Moreira DM, Fleshner NE, Freedland SJ. Baseline perineural invasion is associated with shorter time to progression in men with prostate cancer undergoing active surveillance. REDEEM study. J Urol 2015;194:1258-63.

19. Maru N, Ohori M, Kattan MW, et al. Prognostic significance of the diameter of perineural invasion in radical prostatectomy specimens. Hum Pathol 2001;32:828-33.

20. Rubin MA, Bismar TA, Curtis S, et al. Prostate needle biopsy reporting: how are the surgical members of the Society of Urologic Oncology using pathology reports to guide treatment of prostate cancer patients? Am J Surg Pathol 2004;28:946-52. 